\title{
XLIX. Materials for a history of the prussiates
}

\section{Proust}

To cite this article: M. Proust (1808) XLIX. Materials for a history of the prussiates, Philosophical Magazine Series 1, 32:127, 336-357, DOI: 10.1080/14786440808562804

To link to this article: http://dx.doi.org/10.1080/14786440808562804

$$
\text { 曲 Published online: } 18 \text { May } 2009 .
$$

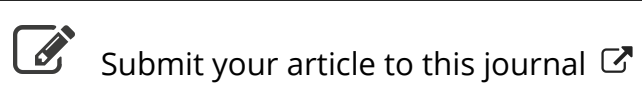

Џll Article views: 2

Q View related articles $\sqsubset$ 
other bandages, with which they are generally tortured in their early years by the active folly of their friends, which, by displacing many of the viscera, impedes their actions, and by compressing them together produces adhesions of one part to another, and affects even the form and aperture of the bones of the pelvis, through which the nascent child must be protruded.

As parturition is a natural, not a morbid process, no medicine should be given, where there is no appearance of disease. The absurd custom of giving a powerful opiate without indication to all women, as soon as they are delivered, is, I make no doubt, frequently attended with injurious, and sometimes with fatal consequences.

Another thing very injurious to the child, is the tying and cutting the navel-string too soon; which should always be left till the child has not only repeatedly breathed, but till all pulsation in the cord ceases. As otherwise the child is much weaker than it ought to be; a part of the blood being left in the placenta, which ought to have been in the child; and at the same time the placenta does not so naturally collapse, and withdraw itself from the sides of the uterus, and is not therefore removed with so much safety and certainty.

[To be continued.]

\section{Materials for a History of the Prussiates.}

By $M$. Proust*.

$T_{\text {He Prussian blue used in commerce is rarely pure. Scheele. }}$ has already noticed this fact. We often find in it, besides alumine, which forms part of it, silex, carbonate, and calcareous sulphate, sulphate of potash, phosphate of iron, the red oxide of this metal, sulphur, oleaginous ammonia, \&c. In order to comprehend the nature of this combination, it is indispensable to use a prussiate without alum, sufficiently washed in the acids and in boiling water. It even appears from a remark of Berthollet, that the prussiate

* Anales de Chimie, tome lx. p. 185. 
of potash may be attached to Prussian blue, so strongly as to resist the washing to a certain degree. I do not think with him, however, that the surplus of this salt should be considered as an-element essential to it, for the Prussian blue which has been well prepared, leaves no trace of saline matter in the residue of its distillation.

Prussian blue, prepared without alum, is cupreous like fine indigo. It loses 0.45 only by combustion. Its residue is red oxide without any mixture of extraneous matters.

Action of the Alkalis.-Prussian blue, when tried with caustic potash, leaves a residue which is only red oxide confounded with alumine. The shade is that of kermes, if the blue be of a good quality; on the contrary, it is pale and earthy if it has been surcharged with alumine; so that we may judge of its nature extremely well from the colour of the residue.

The acids, when applied to a residue properly washed, extract no colour from it: this shows that we may in a single operation rob the Prussian blue of all its acid; but for this purpose it must be finely pulverized, which is extremely difficult to accomplish. If we throw some drops of alkali into water coloured by blue freshly precipitated, the colour is completely discharged : in this case the oxide separated from it does not give the least vestige of colour when we moisten it with an acid. In the process usually followed, it frequently happens that the ochrey residue preserves either some residue of blue which has not been attacked by the alkali, or a mixture of prussiate of potash, and of ferruginous alkaline carbonate, or even these three substances confounded together. I shall now examine two of these cases; the third may easily be guessed at.

If, for instance, we try an acid upon a residue well washed, and which still retains some blue, this last will not be discovered in pulverulent parricles, except in proportion as the acid will free it of yellow oxide. Between this oxide and Prussian blue there is no particular chemical union, as has been hitherto supposed; at least there is nothing which positively indicates that the salino-metallic combination, which we call prussiate of iron, is, like so many others, susceptible

Vol. 32. No. 127. Dec. 1808. 
of a maximum and minimum; and if the mixture of yellow and blue sometimes presented to us by these residues is not green, as might be expected, it is because the yellow oxide always covers these remains of blue in a very great excess; at least I never found the latter to exceed one or two hundredth parts.

I now proceed to the second case. A residue, if well pulverized, may contain no vestiges of blue, but it easily retains the two salts mentioned above. If we apply an acid to it in this state, both give blue in abundance. We shall by and by examine the particular mixture of these two salts; but if it has been carefully washed, the acids can no longer produce any salts from it. Indeed, nothing is more tedious than this washing, for I was obliged to renew the boiling water at least 20 times successively upon a single drachm of residue before I obtained it completely pure: but when we at last succeed, the acids dissolve it without giving blue.

When these residues effervesce with the acids, it is because they contain carbonates of potash or of lime. By washing, the first is carried off; by the application of vinegar after washing, we discover the second. Thus it is not the red oxide which occasions this effervescence: it is not susceptible of being combined with the carbonic acid; it cannot therefore take it from potash in exchange for the prussic acid which it receives from it. In nature, as well as in art, the oxide of iron at the minimum solely may be united with carbonic acid.

One pound of blue of commerce of a fine quality gave nearly nine ounces and a half of crystallized prussiate of potash. It is by no means rare to find in the mother waters, when left to themselves, truncated octahedrons one inch in diameter. When this blue carries sulphuric acid with it, there must be at least four crystallizations, in order to purge the prussiate of the whole sulphate of potash. These mother waters contain alumine sometimes in abundance, sulphate and phosphate of potash, ferruginous alkaline carbonate. By. this we may judge why, in analyses, it is of importance to use crystallized prussiate, and not simple lixiviums of Prussian blue, as formerly made. The prussiate of potash is unalterable 
malterable in dry or humid air : the longest continued cbullition does not aiter its nature in the least: the taste is sweetish, slightly saline, and leaving a sensation of bitterness behind: alcohol does not dissolve it. If we mix some of it with a solution, the prussiate is separated like white flakes of snow, which preserve their lustre when dried, and resembling the silvery kind of gauze presented by the acetate of mercury. When redissolved in water, it reproduces an ordinary solution of triple prussiate.

This salt, which I shall denominate triple to distinguish it from the simple prussiate of potash, is equally constant in its attributes with the most perfect neutral salts. It is of a fine citrine yellow colour, which never leaves it until it changes its state: for this colour, as well as for its two other characteristic properties, of crystallizing and dyeing the red oxide blue, it is indebted to a portion of hlack oxide, which forms an essential part of its constitution. Without this oxide, subjected, like the two other elements of the triple prussiate, to an invariable proportion, this prussiate could in fact neither crystallize, nor form blue with the solutions of iron, the base of which was at the maximum. It is, in short, from this very union that the principle which saturates the potash of the triple salt extracts, as remarked by Berthollet, properties which singularly increase its analogies with the acids.

In this view we may add, that the triple prussiate occupies the middle between the alkaline and the metallic salts. When we reflect, however, upon one property of this salt, which we shall mention presently, it would be difficult to ascertain whether it is to the prussic acid, or to the simple prussiate collectively, that the oxide of iron attaches itself when it is raised to the state of triple prussiate. What is certain, however, is, that we are still ignorant of what aspect or what properies a prussic acid should have, which should be united in precisely a proper proportion with that dose of black oxide, by the assistance of which it can furnish a triple prussiate. On treating the prussic acid with this oxide, we may make Prussian blue, but not the ferruginous kind of acid, which is proper for converting potash into triple salt: this must not be lost sight of ; for we know very well that $\mathrm{Y} 2$ Prussian 
Prussian blue is not of a nature to be combined without a residue with potash. In a word, the triple prussiate, with the exception of its alkaline base, is, if I may so express myself, a compound as to which we have as yet no data, and no idea which authorizes us to consider it rather as a salt, the acid of which would have been particularly exalted by its union with the oxide, than as a combination perfected throughout all its parts by this same oxide.

One property which seems to hinder us from admitting the prussiate as a salt, the acid of which should be exclusively united to the black oxide, is that of its resisting the power of the alkaline hydro-sulphurets. If these re-agents, which spare no other metallic salt, have no action upon the triple prussiate, we are therefore to a certain extent warranted in presuming that the oxide of iron could not well have been exclusively attached to the acid of the triple prussiate, unless we are willing to believe that the affinity of this acid for the oxide is not powerful enough to defend it from the fate which is common to all the other oxides. To conclude: We shall see presently that an affinity so extraordinary, however unexampled it may be in chemistry, is not an impossibility. I shall now proceed to the trial of the hydro-sulphuret of potash upon the triple prussiate.

Hydro-sulphuret and triple Phosphate.-The hydro-sulphuret of potash or of ammonia, even when assisted by heat, has no action upon this salt. If it contained some remains of ferruginous carbonate, it would be freed from it, because the hydro-sulphuret decomposes the latter : we may filter it if necessary, and yet the prussiate still crystallizes under the accustomed form. A similar result leads us to discover, as we originally insinuated, a most particularly intimate com. bination between the three elements of the triple prussiate. But we shall see these same bydro-sulphurets contribute in enabling us to obtain, in all its purity, the white prussiate, or that union in which the iron is at its minimum of oxidation, which I proved in my first memoir upon Prussian blue.

White Prussiate.-On the one hand we must have a flask of green sulphate very much diluted, at the bottom of which we keep some grains of sulphuret of the same metal, 
in order to preserve its base at its minimum. On the other hand, we keep in ebullition over a lamp, a matrass, in which has been placed fifteen or eighteen grains of prussiate of potash and two or three ounces of hydro-sulphureted water : a few seconds after the ebullition or the vapour of it has driven off the air which occupies the vacuum of the matrass, we drop into it some solution of the sulphate: there is a precipitate immediately produced which makes the liquor as white as milk, and which remains so while the beat continues. This is the precipitate which I call white prussiate: this is the prussiate obtained by Fourcroy, Vanquelin, Davy, and all those, without doubt, who, having had regard to the conditions which secure the success of it, have ascertained that the base of the green sulphate could also become the base of a prussiate different from that which has for its base the oxide at the maximum. But as upon passing from one combination to the other the black oxide does not always lose its disposition to be hyper-oxidated, we see that as soon as the matrass is removed from the fire the atmosphere reacts upon the milky-looking mixture, and rapidly exhibits undulations in it which commence by shading it, and which finish by giving it the deep shade of the most perfect blue.

We may also obtain this product in another manner. Drop some grains of prussiate of potash into a very dilute and boiling solution of green sulphate, and we see a precipitate appear, the white of which sustains the action of the air for a short time longer.

The following are a few additional processes, which, if they add nothing to our conviction, are nevertheless interesting from the variety of the means.

Fill two glasses, one with nitrate of iron and the other with green sulphate, both very dilute : afterwards drop into them a crystal of prussiate of potash. In the first we find the crystal is coloured at the same instant of so deep a blue that it resembles black velvet. In the second it crackles, disperses, and falls down in a white powder: but, as before becoming the subjcct of the experiment it had imbibed atY 3

mospheric 
mospheric air, the precipitate resulting from it assumes the appearance of a piece of parsley cheese.

Fill two glasses with boiling water : put a few drops of prussiate into the one, and an equal quantity into the second: add to the latter some drops of hydrosulphuret of potash or of ammonia. These two glasses being thus prepared, drop some nitrate of iron into them : the first yields, as might be expected, a complete blue ; but the second presents the amusing spectacle of a precipitate which, although blue at first, rapidly quits this colour to become white. The theory of these facts is so evident that $I$ shall not dwell upon it, nor shall I repeat all the other experiments mentioned in my first memoir, in order to establish the existence of the two prussiates of iron. If the prussiate at the minimum has no colour when it is not affected by the atmosphere, we find that the green sulphate when dried has none also. The absence of colour in one of these salts is surely not more astonishing than its absence in the other; and finally, if we obtain red oxide by applying the alkalis to the blue prussiates, it is on the contrary black oxide which we extract from the white prussiate. But these differences, which theory previously points out, perfectly coincides with those exhibited by the red and green sulphates under similar circumstances.

In my first memoir I advised the pouring of the prussiate of potash upon the sulphate in a flask, in order to avoid, as much as possible, the mixture of the air, but I succeeded very imperfectly: in the first place because cold liquids always bring some air along with them; and secondly, because I had not thought of sulphuretted hydrogen for purging them. I was not then acquainted with the way in which it acted with these salts.

If, for example, we dilute the solution of green sulphate with three or four times its volume of sulphuric or muriatic acid, the excess of these acids does not in the least change the result. The white prussiate only wanting colour on accomnt of the want of oxygen, we should think that additions like these are not made for the purpose of giving it. Acids 
more concentrated may alter the whiteness of the prussiate, but will never bring it to a perfect blue.

The marine acid, boiled over prussiate also does not make it blue.

This boiling acid is not without action upon the white prussiate. The following is what I remarked on this subject: Some white prussiate is destroyed, some prussic gas disengaged, and we find black oxide in solution: in this case the little Prussian blue formed by the introduction of the air during the interval of the mixtures, predominates over the white, and changes its whiteness to greenish.

The blne prussiate, boiled with the same acid, also gives prussic gas and abandons red oxide, but less is destroyed of it than white prussiate. We may infer from these facts that the muriatic acid, aided by heat, could, strictly speaking, decompose the prussiates, and resume over the prussic its rights as a more energetic acid:-this would not be an astonishing, but it would be at least a tedious, experiment.

Prussiate of Potash and Acids.-Heat marine or weak sulphuric acid in a matrass with crystals of prussiate. When ebullition commences, the gas escapes; let it be received in a bell-glass full of mercury, or burn it by presenting the flame of a candle to it. The flame it emits is variegated red, violet, and yellow: during the dissipation of the gas, the liquor is thickened by the production of a white precipitate, which passes to the blueish. The gas being totally separated, throw the mixture into boiling water, revive with oxygenated muriatic acid; wash and dry the product in a capsule. Four experiments, made at distinct periods, yielded me from 0.34 to 0.35 of complete blue, for a hundred parts of triple prussiate.

Inferences, -100 parts of Prussian blue, without alum, yield 0.55 of red oxide by combustion. This same blue destroyed by the nitric acid also gives 54 . Thus then it is, no doubt, that pure blue of Prussia only contains from 0.54 to 0.55 of red oxide. According to these data, 0.35 parts of blue should render about 0.17 of black oxide, or 0.19 of red oxide. Hence it follows, when we formerly separated the iron of a solution by the prussiate of potash, this salt added 
to the product the 0.19 of red oxide, which resulted from its own decomposition: but the surplus was still greater when, instcad of crystallized prussiate, we used a simple alkaline lixivium of Prussian blue. We shall see the reason of this presently.

When we pass a ley of common potash over Prussian blue, a part of the alkaline carbonate is charged with red oxide; there results a solution which answers to Stahl's martial tincture, and of which pure potash is not susceptible. This solution, which may be also prepared by throwing some drops of nitrate into liquid carbonate, may be mixed with the prussiate of potash without occasioning the least change even by remaining in it. It is this same ferruginous carbonate which, as I have said, is recovered in its mother waters. Now if we add an acid to the mixture of these salts, we precipitate perfect blue from it, because the new solution of oxide which replaces the ferruginous carbonate decomposes in its turn the prussiate of potash, as any solution of iron would do*.

The instant, therefore, that we employ in any analysis a prussic lixivium in place of a crystallized prussiate, we do nothing else than add to the product; in the first place, the red oxide, which formed part of the ferruginous carbonate, then the black oxide which is a constant element of the triple prussiate contained in this lixivium.

Chemists very soon disoovered the vicious effects of these lixiviums, although they did not at first perccive that they contained two very different ferruginous combinations : the carbonate already mentioned, and the triple prussiate. Several experimenters, even when they saw the blue they furnished with the acids, thought this blue was natural, or, in other words, oxide or blue of Prussia; they endeavoured to precipitate it, however, without touching the alkaline prussiate, which they thought to be endowed with the property of dyeing without owing it to the iron. From their efforts arose the recipes for precipitated lixiviums which we find in every book upon chemistry. But since

\footnotetext{
* It is the mixture of these same salts which preparcs the mother waters of soda, for giving Prusian blue when we add an acid to them.
} 
the inquiries of Scheele and Berthollet, we have ascertained that these recipes but imperfectly fulfilled the object in view : for it is easy to see that it was not enough to strip a lixivium of the oxide which the carbonate introduced into it: it still remained to provide against the black oxide which belongs to the triple prussiate, and which we might so much the less suppose to exist, since the addition of the acids, without the intervention of light or of heat could not render sensible the products of its decomposition.

I shall not stop to analyse the phænomena presented during the preparation of the hot or cold lixiviums; because, now that we are well convinced of the inutility of the prussiates with respect to the evaluation of the quantity of iron in analyses, the details are of little interest : for the same reason I pass over the liquid tests proposed with ammonia, chalk, magnesia, \&c., because they are themselves triple prussiates, in which we cannot place confidence, unless we employ them in the same way as the counter-proof proposed by Berthollet. I shall add, only because it is a fact worthy of being recorded in the history of science, that, when the chemist would still take advantage of a lixivium or liquid test, which he had purified by means of an acid, we may be assured he has not attained (as he perhaps flattered himself, the complete separation of the iron ; for it is certain that every lixivium which gives a blue colour with a solution of red oxide, contains also black oxide, sinee without the assistance of the same oxide it could not be dyeing prussiate; or, in other words, every prussiate of potash, which has not been tripled (trisulè) by the black oxide; consequently prussiate of potass, when pure and simple, is not capable of forming blue with a solution where the oxide is at the maximum:this has occurred to those most experienced in analytical processes. It is a truth which Scheele has perfectly established. I repeat, therefore, that the saturated lixiviums or the alkaline prussiates cannot really serve as a dyeing (teignant) reagent unless a portion of black oxide has rendered them triple salts, the red oxide being by no means capable of supplying the place of the black oxide for this purpose. Finally, we may conclude from all these circumstances that the al- 
kaline prussiates or earthy triples should not for the future enter into the class of reagents useful in analyses, for this plain reason, that they cannot discover the iron in any solution, without, at the same moment, adding their own : they ought only to be allowed to make a figure among those which, like turnsole gall-nuts, \&c., are confined to the class of reagents proper for indicating merely if such and such a principle be presented.

Diluted sulphuric acid, when applied to the triple prusviate, furnishes similar results with the muriatic. 100 parts of prussiate restore by this method from 115 to 116 of sulphate of potash. If we knew exactly how much alkali there is in the sulphate, we might infer from the estimate the base of the prussiate of potash. 100 parts of crystals of prussiate lose ten of water by distillation.

In order to complete its decomposition by the acids, the ebullition must be kept up at least half an hour, in order to dissipate the gas entirely, and to obtain the complete separation of the white prussiate which is formed during the operation.

The prussiate of potash is dissolved cold in the muriatic acid, without being decómposed. This mixture requires, according to Berthollet, the assistance of light or of heat.

Vinegar assisted by ebullition also decomposes it: the prussic gas escapes, and the white prussiate is formed; it does not become blueish so rapidly as with the foregoing 2cids : in short, this prussiate, which does not appear except at the very mument when the ternary combination begins to be disorganized, strongly confirms by its whiteness that it is really the oxide at the minimum only, which has the privilege of entering into the formation of the triple prussiate : this is one of those truths which Scheele has completely established. Notwithstanding this, however, the distinction of the oxides in these circumstances is a point to which subsequent chemists have not paid proper attention.

Black Oxide Element of Prussian Blue.-We shall now prove that this oxide, in an invariable dose, is an essential principle in the constitution of the triple prussiate ; but it 
is a point which also deserves some attention, when we see that this same oxide can follow the prussic acid from one combination to the other, without changing its state: when we see also this oxide pass from prussiate to prussiate, return from the latter to the former, circulate through even the most oxidizing medium, without thereby losing the state which constitutes it an oxide at the minimum : this is also a point in the history of prussiates, which in my opinion has not been attended to.

If, for example, it would be correct to say, that without the assistance of the black oxide the prussiate of potash would neither be yellow, crystallizable, nor dyeing, we might assert with equal truth, that the Prussian blue could not be formed without the intervention of this same oxide; and in fact when, with a solution of red oxide of triple prussiate of potash, we make Prussian blue, the black oxide of this salt passes jointly with its acid into the new combination : whence it follows that this oxide, element of the prussiates of potash, becomes so afterwards from the Prussian blue, and even as we shall show from all the other metallic prussiates which are formed with this salt.

This black oxide is found so solidly interwoven in the combination of Prussian blue, so well guarded by its alliance with the prussic acid from all ulterior hyper-oxidation, that we never fail to recover it in this blue, such as it was formerly in the triple prussiate of potash. I shall go further : if we make blue with this prussiate and the green sulphate, the oxide of this last will rise, as we know, to its maximum, in proportion as the blue will be coloured by the impression of the air ; but will it be the same with the black oxide which passes jointly with the acid in Prussian blue? Certainly not. This oxide will not renounce the quality of minor oxide which it had in the prussiate of potash : i.e. if during the exposure to the air the base of the green sulphate, and consequently that of the white prussiate, is raised from 28 to 48 per cent., the black oxide, the inseparable attendant upon the prussic acid, will not participate in this byper-oxidation, it will invariably keep to its 28 per cent.

Not only the atmosphere, which so easily raises to their maximum 
maximum the bases of the sulphate, of the muriate, and of the white prussiate, loses all its activity with respect to the black oxide in question : but still, neither the application of the boiling nitric acid, nor the oxygenated muriatic acid, can succeed in raising the oxidation of this last. These acids may in fact destroy Prussian blue, and even reduce it to red oxide : but while there remains some blue to be destroyed, this last will preserve to the end the black oxide in all its primitive integrity.

If we treat the red oxide with prussic acid, we shall form no kind of combination; - this is conformable to the observation of Scheele : but if we make use of black oxide, we shall obtain greenish prussiate which the air will convert into perfect blue. The black oxide enters therefore into the combination of Prussian blue. If this oxide was not necessary, or if the red oxide could exclusively serve as a base to Prussian blue, we do not see why this oxide, and even its solution, mixed with simple prussiate of potash, would not give Prussian blue.

I have remarked a little higher that the affinity of the prussic acid for this dose of black oxide, which renders it proper for producing triple prussiate, might be powerful enough for saving it from the destiny common to all the oxides which are combined with àcids in general.

It appears to me, in short, that we draw this consequence from the following experiment :

Throw into a flask hydrosulphuret of potash upon Prussian blue, and keep the mixture well corked: in a few days we find the hydrosulphuret converted into triple prussiate, and the red oxide of Prussian blue alone changed into black hydrosulphuret : and hence we see that if the red oxide has followed the example of all the oxides when the hydrosulphuret finds them united to acids, it is not so with the black oxide, which, as we have so frequently said, passes from the triple prussiate of potash into the Prussian blue. This oxide, as it were, forms a separate stripe; it never participates in the changes of which the red oxide is susceptible, which is the base of Prussian blue.

Hydro-sulphuretted water brings the Prussian blue to the state 
state of white prussiate, as it does the red sulphate. This fact I have published in my first memoir, and the power of this reagent never passes beyond; but the bydro-sulphuret of potash totally changes the red and green sulphates into black hydro-sulphureted oxide. Why cannot the hydrosulphuret extend its action to the black oxide in question? There must be a singular affinity, and of which there are few examples in chemistry, which enables the prussic acid, the weakest of the acids in so many respects, to protect this oxide against the whole power of the alkaline hydrosulphurets.

All the metallic solutions which give prussiates with the triple prussiate of potash, certainly follow the example of those of iron. The prussiates resulting from it will preserve in all its purity the black oxide which the prussic acid carries with it. But it is now time to lay before $\mathrm{my}$ readers the grand experiment which demonstrates that Prussian blue is a triple salt, and that the black oxide, which had passed from the triple prussiate of potash into the Prussian blue, may still repass from the Prussian blue into the potash, without having at any moment abandoned its quality of oxide at the minimum.

I presume that this experiment is already anticipated by those who have conceived a clear idea of the nature of the triple prussiate of potash.

Take a Prussian blue, for instance, which has undergone all the reactions which the atmosphere or the most highly oxidating acids may have produced upon it. Apply pure potash to it, and we shall procure a lixivium which will only give triple prussiate, $i$. e. a combination in which we shall find the prussic acid constantly associated with the common dose of black oxide. If this prussiate is really what I have described it to be, which the reader can scarcely doubt, there will be no objection, I think, against the new point of theory which establishes "that the white or blue prussiates are triple combinations, as well as the prussiate of potash which has concurred to their formation."

From the potash of prussiate of manganese there results erystallizable triple prussiate of potash, yellow, and provider 
with all its black oxide. This prussiate of manganese is therefore also a combination tripled (trisulée) by the black oxide : the prussiate of copper, which is of a blood-red colour, is, without doubt, another combination of it, for the simple prussiate of copper is yellow.

Scheele assures us, that other oxides have also the property of trebling (de trisuler) the simple prussiate of potash. It is a course of inquiry so much the more interesting, as it is likely to lead to the discovery of some colour equally precious with that of Prussian blue : and lastly, we may conclude, from all we have seen, that there exists no simple prussiate of iron, a kind of combination of which other metals are nevertheless susceptible, as we shall soon see.

Distillation of Prussian Blue.-This prussiate when exposed to a high temperature is destroyed. It is replaced by new products which confirm the theory given us by Berthollet, upon the nature of the prussic acid. We obtain acid which escapes its destruction, carbonate of ammonia, a little free carbonic acid, and gaseous oxide in abundance : one ounce of the blue of commerce of a good quality gave a little more than two pints and a half of this gas; what was wanting to complete the three pints was carbonic acid. The water of the tub contained prussic acid fixed by ammonia. This prusaiate follows, as we know, the traces of that of simple potash : it cannot form blue with solutions of red oxide; but it gives them with those of oxide at the minimum, because at the same moment it constitutes itself triple or dyeing prussiate.

The residue weighed five drachms 52 grains. It was perfectly black, and answered to the magnet : it is a pyrophorus which rapidly takes fire. If, after having preserved it ill corked so long that it 'cannot take fire of itself, we moisten it with nitric acid at $\mathbf{4 0}$ degrees it burns in a very lively manner. $I$ am inclined to think that in this combustion the iron burns jointly with the charcoal.

If Prussian blue has no alum in it, we find only charcoal and iron in the residue.

The muriatic acid extricates from it with the greatest facility that aromatic hydrogen which announces iron com- 
bined with charcoal. The rest is pure charcoal, one of the elements of the acid destroyed. The two others, the hydrogen and azote, are employed in producing ammonia. As to the carbonic acid and the gaseous oxide, it is equally evident that these are the two oxidations, major and minor, of the charcoal furnished by the oxygen of the two oxides which we have ascertained to exist in Prussian blue.

This decomposition takes place with such a moderate heat that it has appeared to me very convenient when we wished to procure gaseous oxide. There is not the least ground for suspecting any oil to be present : it is very surprising to see that in the course of a destruction where charcoal and bydrogen abound, there is not a single particle of these combustibles which proceeds to constitute itself in any of the respects which can form oil.

The oily and aromatic character assumed by the hydrogen during the solution of the residue, also demonstrates that the combination of iron with charcoal does not require a very high temperature. The charcoal of the blood when it is obtained by a very inferior heat, also contains iron in a carburetted state; for with muriatic acid it also gives aromatic hydrogen. I think Priestley has somewhere noticed the bituminous smell of the hydrngen furnished by the fluxes of charry substances.

Distillation of the triple Prussiate of Potash.-This salt Inses ten per cent of water, and its colour also, for it becomes bleached; it does not become soft without a red heat being applied. Some chemists have thought they discovered in the roasting or flux of it a method of taking away the oxide. The following results will show that these processes lead to nothingr useful :

When this salt enters into fusion, there escapes a little prussic acid, which is seized by the ammonia formed at the same instant. There afterwards passes over a nebulous vapour, which is condensed like farina in the neck of the retort. This vapour is not continued after, when the flux is finished, and the sublimate so formed has the alkaline and bitter taste of the simple prussiate.

Alcohol dissolves a part of it, and what is separated from 
it is triple prussiate without alteration ; $i$. e. the latter gives Prussian blue with the solutions of red oxide, while the other cannot give any.

If we present a lighted candle to the beak of the retort, the prussic acid burns alone, and the carbonic acid proceeding from its combustion forms with ammonia crystals of carbonate, which are condensed in the neck of the retort a few lines above the flame. Let us now pass to the examination of the fused prussiate.

The mass resembles fused sea-salt : it is of an ash-gray, and strongly attracts humidity.

If we taste a piece of it, we do not discover the sweetness of the triple prussiate, but an alkaline taste perfumed with the bitterness of almond kernel supplies the place. This taste announces already, that there is simple prussiate of potash in this residue. Some drops of acid liberate a gas which does not belong to this prussiate, and which suggests the idea that there is carbonate of potash there also.

Lastly, this mass when dissolved, deposits a black powder like mica, and very brilliant. When collected by the filter it is a mixture of charcoal, pure iron, and a little sulphuret of iron. This last is an accidental product : its sulphur proceeds from the decomposition of the sulphate of potash, from which it is difficult to purge the triple prussiate. This powder is attracted by the magnet. A weak acid at first disengages from it sulphureted bydrogen; afterwards comes aromatic hydrogen, and in the last place we only find charcoal powder.

Examination of the Solution of the Residue.-Mix with it a moderate quantity of alcohol at 25 degrees ; there is immediately formed a brilliant kind of snow which may be collected by the filter. When oissolved and crystallized, it gives yellowish sweetish crystals, which, with muriatic acid, furnish prussic acid and white prussiate. Here we bave the prussiate purged from oxide, proposed by M. Richter.

The alcoholic solution is distilled almost dry, it is afterwards covered with alcohol at 30 degrees : one portion of it is then dissolved, and the rest falls to the bottom. The latter when examined is carbonate of potash with some re- 
mains of triple prussiate. The new solution, when distilled, gives simple prussiate, which is known by its taste, and the property of not giving blue with solutions of red oxide. The above are the products which I found after the flux of the triple prussiate of potash.

\section{INFERENCES.}

The triple prussiate cannot support an elevated temperature without being simplified in its composition. It gets rid of the black oxide, and passes to the state of simple prussiate: but the latter can also be reduced to something more simple, as we shall soon see : it then leaves potash in its place, and the ordinary remains of the prussic acid, which are ammonia and charcoal. A part of this last serves for deoxidating the black oxide, to reduce it to iron, and its oxygen into carbonic acid.

During these changes a part of the triple and simple prussiates succeed in subtracting themselves, in proportion, without doubt, as the carbonate makes them into a paste: but it is likely that a long continued high temperature in trong vessels, would at last reduce these prussiates to two binary combinations, which are ammonia and carbonic acid, with potash, iron, and remains of charcoal, which the oxygen of the iron and the humidity was not able to acidify.

Simple Prussiate of Potash.-It is obtained by saturating after Scheele's method potash with prussic gas liberatt $d$ from the prussiates of potash or of mercury ; but it is more expeditiously obtained by keeping the alcohol over a concentrated lixivium of animal charcual. It must be shaken from time to time, and the progress of the solution is ascertained from the alkaline and bitter taste of the alcohol. The lixiviums of charcoal from blood or leather are rarely free from a little hydro-sulphuret, because the sulphate which contaminates the prussiates produces sulphur in them : some then passes into the alcoholic solution; but the charcoal also contributes to it, for I prepared lixiviums with charcoal from blood and very pure carbonate of potash, and yet I found hydro-sulphuret, although in a smaller quantity. It must not be forgotten that sulphur has been already found Vol, 32. No. 127. Dec. 1808. 
in the ammoniacal products of the blood. It even seems that, like phosphorus, it can be fixed in the charcoal, but not in the iron which it contains; for the smell of the aromatic iydromen mentioned above gives no indication of the presence of sulphur.

We easily recognise the simple prussiate by its double alkalino-bitter taste, and by the aroma with which it fills the month. It precipitates in ycllow the solution of copper, and does not give blue with that of red oxide; but it precipitates them in ochrey yellow, as a pure alkali would do*.

Lastly, it gives blue with an ordinary solution of sulphate of iron, because there is at first constituted triple prussiate : afterwards it gives white or blue prussiate of iron. If the prussiate be black, it is because the alkaline hydro-sulphuret introduces hydro-sulphuretted oxide into it ; but we get rid of it with sume drops of acid, and the prussiate of iron appears alone. The simple prussiate cannot be well preserved except in a closed flask. Scheele has shown, that the carbonic acid is sufficient for separating it from the potash while its affinities are feeble; when the black oxide is not united with it, concentraled it refuses to crystallize and runs into a mass, in which, however, we distinguish some saline laminx.

This prussiate is the test liquor proposed by Scheele. Its utility in analysis is not limited, since all solutions the iron in which is at the maximum, (and this is most frequent,) are not, as he has himself shown, by any means sensible to this reagent. In order to employ it carefully, we must bring back to the minimum a part of the oxide of the solutions: this is not always easy, nor without danger of increasing the difficulties of the experiment.

Its Decomposition.-The aqueous solution of this prissiate, on being boiled, abandons a part of its acid : which demonstrates sufficiently that this combination is neither solid

* In a nemoir 1 wrote upon Sigena stone, I announced this union as being. probable; but I now find it was a mistake. A sulphate of iron which I had hyper-oxidated by the nitric acid, aud which, nevertheless, retained some renains of black oxide, deceived me; and scheele, whom 1 contraqicted in this respect, saw more clcarly than I did. 
nor comparable to any of those formed by the oxygenated acids. It froths continually, and has something saponaceous in its appearance. A lighted candle brought near the beak of the retort burns this poriion of acid : but the loss is not confined to this; the acid which this salt retains more strongly, by the help of the potash which begins to predominate, also undergoes by the effects of heat a slow destruction, which converts it into ammonia and carbonic acid. At whatever period of the ebullition we take the product, we always find carbonate of ammonia in it mixed with a little prussic acid; and latterly, when the water begins to disappear, this carbonate is condensed in spiculæ in the neck of the retort.

If we add more water in order to continue the ebullition, these same products are found in the water of the receiver. But after four or five successive and similar distillations, we cease to perceive them, although the saline residue still contains prussic acid in a sensible degree.

It must then be dissolved with alcuhol: part of it is dissolved, and the other totally resists its action. In the alcoholic liquor we in fact find prussiate of potash, but the saline mass undissolved is nothing else but carbonate of potash. The object of the two following experiments was to remove all doubt as to the destruction of the simple prussiate by the heat of ebullition alone.

This prussiate does not disturb the muriate of lime: but that which has undergone a long ebullition precipitates it abundantly in calcareous carbonate. There is a transformation therefore of prussiate into carbonate of potash.

Two quantities of prussiate, the one altered by a long ebullition, and the other entire, were employed to precipitate ordinary sulphate of iron. Both gave a blue colour; but after being revived, the former quantity occupied three times Iess room than the latter.

If we make dry simple prussiate red-hot, there exhales carbonate of ammonia accompanied by an oleaginous vapour resembling that of hartshorn. The saline mass when dissolved, separates charcoal, and it is still carbonate of potash mixed with some indecomposed prussiate. 
INFERENCES.

All these results certainly bear us out in the conclusion, that the simple prussiate of potash is, as Scheele has already discovered, a fragile combination, the elements of which are as easily separated as those of complex conbinations. We find, in short, that a part of the acid is removed from the potash by the sole force of dilatation; while the other part, which is longer subjected to the action of caloric, is destroyed in order to be changed into ammonia and carbonic acid. Let us now make the application of this conclusion.

The triple prussiate of potash undergoes no derangement by repeated ebullitions. The lixiviums employed in the manufacture of Prussian blue contain, as we shall presently see, triple prussiate and simple prussiate. Besides, there is no ammoniacal salt in them. We are of opinion, that the great excess of carbonate of potash which they contain would not admit of their presence; but they exhale ammonia while in a state of ebullition. From whence could this ammonia proceed, if it was not from the decomposition of the simple prussiate? We may therefore conclude, that the boiling or concentration of the lixiviums exposes them to be deteriorated by the destruction of this same prussiate, which we cannot too much preserve : and as the carbonate of potash is also one of the principal result $x$ of this destruction, it does not cease to add to what is already there.

Curadeau was well aware of the deterioration which the boiling of the lixiviums produces, and he very happily prevented the bad effects by adding a little sulphate of iron to them. This is conformable to Scheele's principle, who has shown that the simple prussiate changes into triple prussiate as soon as it can be associated with a portion of black oxide, and thereby be guarded against decomposition. As to the products from the destruction of the prussiate by fusion or by ebullition, there is certainly nothing extraordinary in them, since it is sufficient to know the nature of the prussic acid in order to prevent them ; but it is not so with respect to the carbonic acid which is presented during one of these destructions. Whence comes the oxygen, for example, which, during the ebullition of the aqueous prussiate, succeeds 
ceeds in acidifying the charcoal of the prussic acid? Either this uxygen will be, like hydrogen, azote and carbon, one of the constituents of the prussic, and which is destroyed; or it must be supposed that there is a decomposition of water. It is not time yet, as I think, to choose between these tivo opinions; but until we have some more light on the subject, I shall say that if we reflect on the circumstances accompanying the production of the prussic acid, we shall adopt in the nean time the opinion of Berthollet in preference to every other hypothesis. "It appears difficult," says that author, "to suppose the existence of oxygen in a substance which contains elements so disposed to form peculiar combinations witb it, as hydrogen and carbon are, and which can frequently support so great a degree of heat, without undergoing lecomposition." In truth, in order to admit that this acid is an oxygenated product, we ought to suppose that such an acid is capable of disputing the oxygen with the carbon which envelops it on all hands, and we cannot do less than place it at the head, not of the acids, but of the oxides, which are known to be the must 'difficult of reduction.

\section{Some Account of a remarkable Case of Tetanus*. S. C., æt. 22, thin and cielicate, a glass-cutter by trade,} says, that he accidentally trod on a nail which penetrated the bottom of his foot about half an inch.-It inflamed, continued painful two or three days, and then healed without further trouble.

Nine days after the puncture, he was attacked with pain in his bowels, with frequent and copions evacuation by stool. This complaint continued till the next day at noon, when it ceased, but left him much weakcned by the purging.

11 th day.-Still sensible of weakness. In the evening returning from his work be grot wet through his clothes.

12th day.-Obliged to put on his wet clothes in the morning; and in returning to his work was again drencher with

* Communicated by John Taunton, Esq.

Z 3

rain. 